\title{
The Curved MCA: Influence of Vessel Anatomy on Recanalization Results of Mechanical Thrombectomy after Acute Ischemic Stroke
}

\author{
B.J. Schwaiger, A.S. Gersing, C. Zimmer, and S. Prothmann
}

\begin{abstract}
BACKGROUND AND PURPOSE: Vessel anatomy is assumed to influence results of endovascular mechanical thrombectomy using stent retrievers. The purpose of this study was to analyze the influence of vessel curvature on recanalization results in patients with acute ischemic stroke caused by large-vessel occlusion.
\end{abstract}

MATERIALS AND METHODS: In 159 patients (70 \pm 12.8 years of age; 79 women) treated for acute ischemic stroke after carotid T and/or MCA occlusion by using stent retrievers, the following angles were measured in standard anteroposterior angiograms to describe vessel anatomy: first, between the terminal ICA segment and the most downward curved Ml segment conterminous to the proximal face of the thrombus (ICA/Ml angle); second, between the most proximal Ml segment and the segment immediately conterminous to the thrombus (M1/M1 angle); and additionally, in patients with distal M1/proximal M2 occlusion, the angle of the last curvature proximal of the thrombus (M1/M2 angle). Angles of patients with-versus-without successful recanalization were compared.

RESULTS: Patients without successful recanalization (TICI $0-2 a$ ) showed significantly larger ICA $/ \mathrm{Ml}$ angles (mean, $110^{\circ} \pm 23.8^{\circ}$ versus $69^{\circ} \pm$ $\left.28.7^{\circ}, P<.001\right)$ and significantly larger $\mathrm{Ml} / \mathrm{Ml}$ angles $\left(56^{\circ} \pm 29.2^{\circ}\right.$ versus $\left.29^{\circ} \pm 26.6^{\circ}, P=.001\right)$ than patients with successful recanalization ( $\mathrm{TICl} 2 \mathrm{~b} / 3$ ). In patients without successful recanalization after a distal M1 or proximal M2 occlusion, the M1/M2 angle was significantly larger than that in patients with successful recanalization $\left(117^{\circ} \pm 34.3^{\circ}\right.$ versus $\left.67^{\circ} \pm 29.5^{\circ}, P=.006\right)$.

CONCLUSIONS: This retrospective analysis showed that mechanical thrombectomy in the anterior circulation was significantly less often successful in patients with large vessel angles. Therefore, vessel curvature significantly influences the results of mechanical thrombectomy with stent retrievers for treatment of acute ischemic stroke. Further work is needed to understand the underlying causality.

ABBREVIATIONS: MT = endovascular mechanical thrombectomy; ROC = receiver operating characteristic

A cute ischemic stroke is one of the leading causes of morbidity and mortality in industrialized countries. ${ }^{1}$ While studies suggest that intravenous thrombolysis by using recombinant tissue plasminogen activator is barely effective in large-vessel occlu-

Received September 30, 2014; accepted after revision November 17.

From the Department of Diagnostic and Interventional Neuroradiology, Klinikum rechts der Isar der Technischen Universität München, Munich, Germany.

Preliminary results previously presented as part of the following contribution to the World Federation of Neuroradiological Societies Symposium Neuroradiologicum, September 7-12, 2014; Istanbul, Turkey: Schwaiger BJ, Kober F, Gersing AS, et al. "The pREset Retriever for Endovascular Treatment of Stroke after MCA Occlusion: Safety and Clinical Outcome."

This work has been performed in accordance with the standards defined by the local ethics committee and the standards laid down in the 1964 Declaration of Helsinki and its later amendments.

Please address correspondence to Benedikt J. Schwaiger, MD, Department of Diagnostic and Interventional Neuroradiology, Klinikum rechts der Isar der Technischen Universität München, Ismaninger Str 22, 81675 Munich, Germany; e-mail:

benedikt.schwaiger@tum.de

http://dx.doi.org/10.3174/ajnr.A4222 sions, ${ }^{2-4}$ recent studies have shown that endovascular mechanical thrombectomy (MT) by using second-generation devices, known as stent retrievers, is associated with high recanalization rates and substantially improved clinical outcome. ${ }^{5-11}$ However, in approximately $10 \%-25 \%$ of the patients, a successful recanalization (TICI 2b/3) still cannot be achieved. ${ }^{5-14}$ Only a couple of technical factors are understood that may determine the fate of these patients: First, histologic characteristics of thromboemboli may influence recanalization results. ${ }^{15}$ Second, thrombus length has been identified as a relevant factor. ${ }^{16} \mathrm{~A}$ recent study presented contradictory results, reporting that the recanalization success of MT was not significantly influenced by clot length. ${ }^{17}$ The exact thrombus location, being more proximal or distal within the MCA M1 segment, was demonstrated to be a significant determinant for clinical outcome, however not for recanalization success. ${ }^{18}$

To date, no evidence of vessel anatomy influencing recanalization success exists, though experienced interventionalists report that MT with stent retrievers is less likely to be successful in 
patients with strongly curved vessels. Zhu et al ${ }^{19}$ reported that vessel branching and curvature are important determinants of recanalization success; however, their work was based on indirect MR imaging findings and focused on thrombectomy by using the Merci retriever (Concentric Medical, Mountain View, California), a first-generation device, which is now obsolete in most cases. In a previous study on MT by using the pREset thrombus retriever (Phenox, Bochum, Germany), we observed that successful recanalization was significantly less likely in patients with strongly curved MCAs. ${ }^{13}$ Consequently, in this study, we analyzed the influence of vessel anatomy, assessed in anteroposterior angiograms, on recanalization results in a larger sample size. The purpose of this study was also to assess information on prognostic cutoff values and the prevalence of unfavorable MCA anatomy.

\section{MATERIALS AND METHODS}

\section{Patients, Therapy Strategy, and CT}

In the stroke data base of our institution, we identified patients treated for symptomatic acute ischemic stroke after unilateral occlusion in the anterior circulation by using stent retrievers between July 2011 and April 2014. We included 159 patients (mean age, $70 \pm 12.8$ years; 79 women) presenting with 1 of the following occlusion types: short-segment carotid T (ICA terminus including proximal MCA) occlusion encompassing only the terminal ICA segment, isolated M1 segment occlusion, combined occlusion of the M1 and the proximal M2, or isolated proximal M2 occlusion.

Patients with proximal and long-segment ICA occlusion, isolated anterior cerebral artery occlusion, or occlusion of $>1$ intracranial vessel were excluded as well as patients with other vascular pathologies such as pre-existing ICA or intracranial stenosis or dissection, suspected vasculitis, Moyamoya disease, or acute ischemic stroke caused by an endovascular procedure. Patients in whom the occlusion site could not be reached due to proximal vessel anatomy were excluded from the analysis as well.

There were no exclusion criteria regarding the number of devices used and passages of the thrombus or any demographic or clinical parameters.

A neurologic deficit of an NIHSS score of $\geq 4$ was required to indicate treatment, following the standard operating procedure of our institution. The NIHSS score was assessed at admission for baseline evaluation of the patient's clinical status.

Preinterventionally, IV rtPA was applied within a timeframe of 4.5 hours after observed symptom onset if no contraindications existed, following the guidelines by the German Society of Neurology. ${ }^{20}$ Patients received a dose of 0.9 -mg rtPA per kilogram of body weight. Ten percent of the total dose was applied as an immediate loading dose; then, the remaining 90\% was applied during the following hour, regardless of the starting time of angiography and MT.

MT was performed in patients who presented within a timeframe of 6 hours after symptom onset. Contraindications for MT were intracranial hemorrhage and infarct demarcation of more than onethird of the MCA territory in the most recent CT. Patients with wake-up stroke were included if they presented within 3 hours after discovery and only if there was no infarct demarcation detected by noncontrast CT and there was an MTT/CBV mismatch of $>30 \%$ as detected by CT perfusion, according to the standard operating procedure of our institution and recent literature..$^{21-23}$
Time durations were measured from symptom onset to groin puncture, from symptom onset to reperfusion or the end of the procedure in case of unsuccessful recanalization, and from groin puncture to reperfusion or the end of the procedure, respectively.

All patients underwent CT either on a $64-$ or 256-detector row CT scanner (Brilliance 64/iCT; Philips Healthcare, Best, the Netherlands) immediately at admission, to rule out hemorrhage or infarct demarcation. In addition, CT angiography was performed to verify the occlusion type and to analyze vessel patterns. A further CT or MR imaging was performed routinely within 24 hours after endovascular treatment or in case of any clinical deterioration. The complete imaging data including angiograms were re-evaluated for this study by 2 neuroradiologists in a consensus reading (S.P. and B.J.S.).

\section{Endovascular Treatment and Evaluation}

Depending on patient compliance and vigilance, procedures were performed with the patient under either conscious sedation or general anesthesia. An 8F guiding catheter (VISTA BRITE TIP; Cordis, Fremont, California) for cervical access was placed through a short $8 \mathrm{~F}$ sheath after the femoral artery was punctured. By a 3-axial approach, a 0.054-inch intermediate catheter (5MAX; Penumbra, Alameda, California) containing a 0.021-inch microcatheter (Trevo Pro 18; Stryker, Kalamazoo, Michigan) and a 0.014-inch microwire (Traxcess 14; MicroVention, Tustin, California) was advanced intracranially. The occlusion site was passed with the microwire and the microcatheter, which was placed as close as possible to the proximal thrombus face. In this position, the stent retriever was deployed, covering the proximal and the distal thrombus borders. After a delay of 5 minutes, the device was retrieved under constant manual aspiration into the intermediate catheter. This procedure was repeated until successful recanalization was achieved or it was aborted in case of excessive procedural time and negligible chances for success. In this study, the following stent retrievers were used either as a sole device or in combination with other devices, according to the performing interventionalist's individual decision: Solitaire FR Revascularization Device (Covidien, Irvine, California), Trevo and Trevo ProVue Retriever (Stryker), pREset thrombus retriever, Capture LP (MindFrame, Irvine, California), Aperio Thrombectomy System (Acandis, Pforzheim, Germany), and Separator 3D (Penumbra).

Recanalization results were graded in the final angiogram by using the TICI score. TICI $2 \mathrm{~b}$ and 3 scores were considered successful recanalization.

Perforation, dissection, thrombus dispersion, and focal or diffuse $\mathrm{SAH}$ were determined as procedure-related complications. Stroke-related hemorrhagic events (hemorrhagic infarction and parenchymal hematoma) were characterized separately, according to the methods used in the European Cooperative Acute Stroke Study trials. ${ }^{24}$ Complications and hemorrhage were defined as clinically relevant if they were associated with an NIHSS increase of $\geq 4$ points.

\section{Vessel Anatomy Evaluation}

DSA was performed on a biplanar Allura Xper FD scanner (Philips Healthcare). For assessment of vessel anatomy, standard 


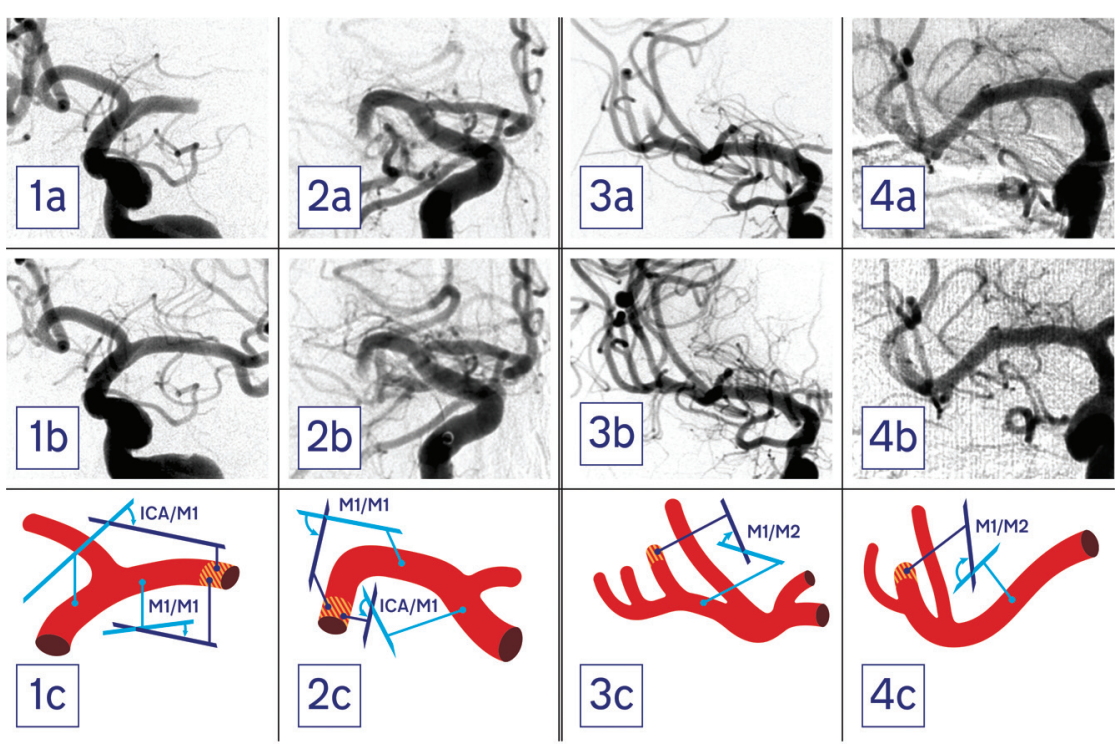

FIG 1. First and second columns (patients 1 and 2 ) show pre- $(a)$ and postprocedural ( $b$ ) angiograms of patients with a proximal M1 occlusion as well as schematics of the vessel anatomy (c). The thrombus site is hatched red and yellow. In patient 1, the ICA/MI was $53^{\circ}$ and the $\mathrm{Ml} / \mathrm{Ml}$ was $18^{\circ}$, and he was successfully recanalized (TICI 3). Patient 2 presented with an ICA/MI of $140^{\circ}$ and an $\mathrm{MI} / \mathrm{Ml}$ of $105^{\circ}$. In this patient, thrombectomy was unsuccessful ( $\mathrm{TICl} 0$ ). Third and fourth columns (patients 3 and 4 ) show pre- $(a)$ and postprocedural $(b)$ angiograms of patients with a proximal M2 occlusion as well as vessel schematics (c). While patient 3 presented with an $\mathrm{Ml} / \mathrm{M} 2$ angle of $51^{\circ}$ and was successfully recanalized ( $\mathrm{TICl} 3$ ), in patient 4 , an $\mathrm{M} 1 / \mathrm{M} 2$ angle of $128^{\circ}$ was measured and thrombectomy was unsuccessful ( $\mathrm{TICl} 1$ ).

Table 1: Epidemiologic, clinical, and technical parameters for all patients and a comparison between subgroups, depending on recanalization success

\begin{tabular}{|c|c|c|c|c|}
\hline Parameter & All Patients & $\mathrm{TICl} 0-2 \mathrm{a}$ & $\mathrm{TICl} 2 \mathrm{~b} / 3$ & $P$ Value \\
\hline Group/subgroup size & 159 & $24(15.1 \%)$ & $135(84.9 \%)$ & \\
\hline Female sex (No., \% of subgroup) & $79(49.7 \%)$ & $11(45.8 \%)$ & $68(50.4 \%)$ & .825 \\
\hline Age (yr, mean) & $70 \pm 12.8$ & $70 \pm 10.7$ & $70 \pm 13.2$ & .964 \\
\hline Wake-up (No., \% of subgroup) & $30(18.9 \%)$ & $3(12.5 \%)$ & $27(20 \%)$ & .572 \\
\hline Time symptom onset to puncture (min, mean) ${ }^{a}$ & $230 \pm 80.0$ & $232 \pm 82.6$ & $230 \pm 79.9$ & .902 \\
\hline Procedure time (min, mean) ${ }^{\mathrm{b}}$ & $55 \pm 35.1$ & $69 \pm 38.2$ & $53 \pm 34.3$ & .058 \\
\hline Time onset to recanalization (min, mean) ${ }^{c}$ & $285 \pm 86.9$ & $303 \pm 96.7$ & $283 \pm 85.4$ & .349 \\
\hline NIHSS score at admission (median, range) & $15(4-27)$ & $16(7-24)$ & $14(4-27)$ & .198 \\
\hline IV rtPA administered (No., \% of subgroup) & $112(70.4 \%)$ & $15(62.5 \%)$ & $97(71.9 \%)$ & .345 \\
\hline General anesthesia applied (No., \% of subgroup) & $127(79.9 \%)$ & $20(83.3 \%)$ & $107(79.3 \%)$ & .787 \\
\hline Passages of device (No., median, range) & $2(1-12)$ & $4(1-10)$ & $2(1-12)$ & $.008^{d}$ \\
\hline$<1$ Device used (No., \% of subgroup) & $55(34.6 \%)$ & $14(58.3 \%)$ & $41(30 \%)$ & $.011^{\mathrm{d}}$ \\
\hline
\end{tabular}

a Data missing for 24 patients.

${ }^{\mathrm{b}}$ Data missing for 9 patients.

c Data missing for 21 patients.

${ }^{\mathrm{d}}$ Significant at $P<.05$.

anteroposterior projections of the final angiograms were analyzed (Fig 1).

Patients with short-segment carotid $\mathrm{T}$ occlusion and proximal M1 occlusion were summarized as having proximal occlusion, while patients with terminal M1 or proximal M2 occlusion were summarized as having distal occlusion. Patients with combined proximal and distal occlusions were assigned to both subgroups.

In patients with a proximal occlusion, we measured 2 angles: first, the angle between the terminal ICA segment and the most downward bent M1 segment (Fig 1, subsequently referred to as the ICA/M1 angle); and second, the angle between the most proximal M1 segment emerging from the carotid T and the M1 segment immediately conterminous to the proximal thrombus face (M1/M1 angle).

In patients with a distal occlusion, in addition to these 2 angles, a third angle was measured between the vessel segment conterminous to the occlusion site and the segment immediately proximally adjacent to this (M1/ M2 angle).

In patients with carotid $\mathrm{T}$ occlusion and M1 segment occlusion immediately adjacent to the carotid $\mathrm{T}$, angle measurements were not possible in preinterventional angiograms due to low vessel contrast. Therefore, the frame with the best vessel contrast was selected from the final control angiogram. Thus, correct measurements were possible even in patients with incomplete recanalization after carotid T occlusion $(n=3)$ or M1 segment occlusions adjacent to the carotid $\mathrm{T}(n=7)$ due to partially re-established vessel contrast in all cases.

All measurements were performed by using standard PACS tools.

\section{Statistics}

In addition to descriptive statistics, the Fisher exact test was used for categoric data and the exact Mann-Whitney $U$ test, for nonparametric testing. The Student $t$ test was used for continuous data. To analyze the influence of the measured angles on the recanalization results, we used logistic regression models, correcting for several covariates. Receiver operating characteristic (ROC) analyses were performed, and the areas under the ROC analysis curves were used to evaluate the diagnostic performance of the measured angles to differentiate patients with-versus-without successful recanalization. The Youden $J$ statistic was used to identify the optimal cutoff values for differentiation of patients. ${ }^{25}$ Correlations were analyzed by the Pearson $r$. Statistical analyses were performed by using SPSS, Release 22 (IBM, Armonk, New York) and were supervised by a statistician.

\section{RESULTS}

One hundred fifty-nine patients (mean age, $70 \pm 12.8$ years; 79 women) were included in this study (Table 1). Angle measurements were feasible in final control angiograms of all selected patients, including those with carotid T occlusions.

The median NIHSS score at admission was 15 (range, 4-27). Thirty patients (18.9\%) were admitted after presenting with a wake-up stroke with an unknown time from symptom onset to reperfusion, while in the remaining patients, the mean time between observed symptom onset and groin puncture was $230 \pm$ 80.0 minutes. In the latter, the exact time was not documented in 21 cases $(16.0 \%)$, but it definitely ranged within a time window of 
Table 2: Angles, $P$ values, AUCs for ROC analyses, and optimal cutoff values to differentiate TICl subgroups and Youden indices for different vessel angles ${ }^{\mathrm{a}}$

\begin{tabular}{|c|c|c|c|c|c|c|c|}
\hline Occlusion Pattern & $\begin{array}{l}\text { Group } \\
\text { Size }\end{array}$ & Angle & $\begin{array}{l}\mathrm{TICl} 0-2 \mathrm{a} \\
\text { (mean) }\end{array}$ & $\begin{array}{c}\mathrm{TICl} 2 \mathrm{~b} / 3 \\
\text { (mean) }\end{array}$ & $P$ & ROC AUC & $\begin{array}{l}\text { Cutoff and } \\
\text { Youden index }\end{array}$ \\
\hline \multirow[t]{2}{*}{ Proximal (carotid T or proximal Ml segment) } & 125 & ICA/MI & $110 \pm 23.8$ & $69 \pm 28.7$ & $.000^{\mathrm{b}}$ & $.872^{\mathrm{d}}$ & $100^{\circ}(V=0.69)$ \\
\hline & 125 & $\mathrm{Ml} / \mathrm{Ml}$ & $56 \pm 29.2$ & $29 \pm 26.6$ & $.001^{\mathrm{b}}$ & $.759^{d}$ & $\left.36^{\circ} \mathrm{U}=0.40\right)$ \\
\hline Distal (distal M1 or proximal M2 segment) & 36 & $\mathrm{M} 1 / \mathrm{M} 2$ & $117 \pm 34.3$ & $67 \pm 29.5$ & $.006^{\mathrm{c}}$ & $.865^{d}$ & $97^{\circ}(U=0.64)$ \\
\hline
\end{tabular}

Note:-AUC indicates area under the curve.

a $P$ values significant at $P<.05$.

${ }^{\mathrm{b}}$ Corrected for age, sex, and IV rtPA (yes/no).

Corrected for age.

d Significant at $P<.005$.

6 hours. In addition, no infarct demarcation was shown on the CT scan immediately before intervention in these patients.

A successful recanalization (TICI 2b/3) was achieved in 135 patients $(84.9 \%)$ after an average procedure time of $55 \pm 35.1$ minutes. TICI $2 \mathrm{~b} / 3$ was achieved in 29 patients $(90.6 \%)$ with a carotid T occlusion, in 109 patients $(87.2 \%)$ with a proximal M1 occlusion, and in 28 patients $(77.8 \%)$ with a distal $\mathrm{M} 1$ or proximal M2 occlusion.

Stent retrievers were used in the following cases: pREset in 91 (57\%), Solitaire in 57 (36\%), Trevo and Trevo ProVue in 18 (11\%), and others (Capture LP, Aperio, and Separator 3D) in 13 (8\%).

MT was performed with the patient under conscious sedation in 32 patients $(20.1 \%)$ and general anesthesia in 127 patients (79.9\%). No significant influence of the type of anesthesia on the recanalization results was found $(P>.05)$. Patients undergoing general anesthesia had significantly higher NIHSS values than patients treated under conscious sedation (median, 16; range, 4-27 versus $11,5-22 ; P<.001)$.

Between subgroups of patients with-versus-without successful recanalization, there were no significant differences in demographic parameters, NIHSS score at admission, time between symptom onset and groin puncture, and procedure time. During MT or at follow-up imaging, we detected the following procedure-related complications: dispersion of the thrombus in 6 patients $(3.8 \%)$, periprocedural perforation of the vessel in 4 patients $(2.5 \%)$, and dissection of a vessel in 4 patients $(2.5 \%)$. A focal SAH was found in 5 patients $(3.1 \%)$, while diffuse SAH was not found in any patients. According to the European Cooperative Acute Stroke Study classification for hemorrhagic events, there were 10 patients $(6.3 \%)$ with small petechial hemorrhagic infarction, 3 patients (1.9\%) with more confluent petechial hemorrhagic infarction, 5 patients $(3.1 \%)$ with a parenchymal hematoma with a mild space-occupying effect, and 3 patients $(1.9 \%)$ with a parenchymal hematoma with a significant space-occupying effect. This latter parenchymal hematoma only occurred in patients with large MCA territory infarctions after hemicraniectomy. None of the described procedure-related complications or hemorrhagic events were associated with an additional relevant clinical deterioration. There were no significant differences between patients with-versus-without successful recanalization regarding complication rates and hemorrhagic events $(P>.05)$.

One hundred twenty-five patients were assigned to the subgroup with proximal occlusions. In patients without successful recanalization (TICI $0-2 \mathrm{a}$ ), the ICA/M1 angle was significantly larger than that in patients with successfully recanalization after correction for age, sex, and IV rtPA application (mean, $110^{\circ} \pm$ $23.8^{\circ}$ versus $69^{\circ} \pm 28.7^{\circ} ; P<.001$; Table 2 ). For differentiating these patients regarding recanalization success, an area under the receiver operating characteristic analysis curve of $0.87(P<.001)$ was computed for an optimal cutoff of the ICA/M1 angle at $100^{\circ}$ (sensitivity, 0.81; specificity, 0.88; Youden $J=0.69$ ). By analogy, the M1/M1 angle also differed significantly between patients without-versus-with successful recanalization after correction for age, sex, and IV rtPA application (mean, $56^{\circ} \pm 29.2^{\circ}$ versus $29^{\circ} \pm$ $\left.26.6^{\circ} ; P=.001\right)$. For the M1/M1 angle, ROC analysis provided the corresponding area under the curve of $0.76(P=.001)$, with an optimal cutoff at $36^{\circ}$ (sensitivity, 0.75 ; specificity, 0.65 ; Youden $J=0.40)$.

Between the ICA/M1 angle and the M1/M1 angle, a significant correlation was found $(r=0.54, P<.001)$.

Thirty-six patients were assigned to the subgroup with distal occlusions. In patients without successful recanalization (TICI $0-2 \mathrm{a})$, the M1/M2 angle was significantly higher than that in patients with successful recanalization after correction for age (mean, $117^{\circ} \pm 34.3^{\circ}$ versus $67^{\circ} \pm 29.5^{\circ} ; P=.006$; Table 2 ). Using ROC analysis, we found an area under the curve of $0.87(P=.001)$ for the ICA/M1 angle, with an optimal cutoff at $97^{\circ}$ (sensitivity, 0.78; specificity, 0.86; Youden $J=0.64$ ).

In this subgroup with distal occlusion, no significant differences were found between patients with-versus-without successful recanalization regarding the proximal angles ICA/M1 and M1/M1 $(P>.05)$.

After defining an ICA/M1 angle of $>100^{\circ}$ as a curved MCA, 26 of 125 patients with proximal vessel occlusion were detected (20.8\%; mean age, $70 \pm 10.4$ years; 10 women) who presented with this curved vessel anatomy. In these patients, TICI $2 \mathrm{~b} / 3$ was significantly less often achieved than in patients with a lower ICA/M1 angle (50.0\% versus $97.0 \%, P<.001)$.

In the subgroup of patients with distal occlusion, 11 of 36 (30.6\%; mean age, $69.82 \pm 14.2$ years; 3 women) had an M1/M2 angle of $>97^{\circ}$. Compared with patients with lower M1/M2 angles, TICI $2 \mathrm{~b} / 3$ was significantly less often achieved in these patients (36.4\% versus $92.0 \%, P=.001$ ).

There were no significant differences regarding demographic parameters between patients with curved $\left(>100^{\circ} \mathrm{ICA} / \mathrm{M} 1\right)$ and straight $\left(<100^{\circ} \mathrm{ICA} / \mathrm{M} 1\right)$ proximal vessel angles as well as between patients with curved $\left(>97^{\circ} \mathrm{M} 1 / \mathrm{M} 2\right)$ and straight $\left(<97^{\circ}\right.$ $\mathrm{M} 1 / \mathrm{M} 2)$ distal vessel angles.

For the comparison of different stent retrievers, the seldomused devices, Capture LP, Aperio, and Separator 3D, were grouped as "others," while the most frequently used devices, Solitaire FR, pREset, and Trevo/Trevo ProVue were assessed sepa- 
rately. We analyzed whether one of the devices was used more frequently in patients who were successfully recanalized despite the presence of curved vessels. None of the devices were significantly over-represented in either subgroup $(P>.05)$. In the whole study population, none of the devices were associated with a significantly better recanalization rate or a significantly elevated complication rate $(P>.05)$. In patients without successful recanalization, significantly more passages were performed and $>1$ device was deployed significantly more often than in patients with successful recanalization $(P=.008$ and $P=.011$, respectively).

A strong vessel curvature $\left(\mathrm{ICA} / \mathrm{M} 1>100^{\circ}, \mathrm{M} 1 / \mathrm{M} 1>36^{\circ}\right.$, $\mathrm{M} 1 / \mathrm{M} 2>97^{\circ}$, respectively) was associated with neither a significantly higher rate of procedure-related complications such as thrombus dispersion, vessel perforation, or dissection nor hemorrhagic events $(P>.05$, respectively).

In patients with successful recanalization, IV rtPA was not administered significantly more often than in patients with TICI $0-2 \mathrm{a}(71.9 \%$ in TICI $2 \mathrm{~b} / 3$ versus $62.5 \%$ in TICI $0-2 \mathrm{a} ; P>.05)$.

\section{DISCUSSION}

In this study, we have shown that vessel anatomy, in particular the curvature of the carotid T and the MCA M1 and M2 segments, has a significant influence on the technical results of MT by using stent retrievers. Patients without successful recanalization (TICI 0-2a) had both significantly larger ICA/M1 and M1/M1 angles in proximal vessel occlusions and significantly larger M1/M2 angles in distal vessel occlusions.

To our knowledge, this phenomenon has not been described in detail before. In patients with acute ischemic stroke after MCA occlusion, Zhu et $\mathrm{al}^{19}$ assessed the clot configuration by using gradient-echo MR images in axial sections and thus the vessel anatomy within the occlusion site. However, this method provides only indirect information on the vessel curvature, and vessel anatomy proximal to the occlusion site cannot be analyzed.

The relevant geometric details provided by the MCA segment are direction changes toward superior and inferior. Therefore MCA angles are visualized and assessed best in anteroposterior projections.

Furthermore, per se, vascular structures are shown in DSA most precisely; however, only vessel sections passed by intra-arterial contrast agent are visible. Therefore, a limitation of the technique used is that we were only able to measure angles including the most distal vessel segment visualized that was immediately conterminous to the occlusion site. Clot configuration along its entire length and vessel anatomy within the occlusion site could not be analyzed; thus, its influence on recanalization success remains unclear. Further analyses may make use of the microwire passage as an auxiliary marker for vessel anatomy within the occlusion site, or, more directly, of 3D susceptibility-weighted imaging data from patients undergoing MR imaging as a primary stroke imaging technique.

While vessel anatomy assessment based on anteroposterior projections remains an approximation, the most precise angle measurement may be possible in 3D models obtained from CTA. For this analysis, this method has been tested in a small group of patients; however, it proved to be impractical due to the compar- atively low spatial resolution and the poor vessel contrast immediately adjacent to the occlusion site.

Because this is a retrospective analysis, only assumptions can be made regarding the causal relation between vessel anatomy and successful recanalization. First, when retracting the stent retriever toward the distal-access catheter following a strongly curved vessel segment, the stent-like device may diminish its full spatial extension and thus the grip and interacting forces on the thrombus may be reduced, an effect that has been described as "tapering." ${ }^{2,13}$ Second, strongly curved vessels may increase friction among the vessel walls, catheters, wires, and device, thus impeding passage and retrieving maneuvers. Third, during the retrieving maneuver, one may observe a change of the vessel anatomy: When applying traction to the pusher wire to which the stent retriever is connected, the expanded device transfers this tension to the vessel anatomy, causing both a folding of vessel segments proximal and an elongation distal to the deployment site. This effect may be more prominent in initially already strongly curved vessels and may increase angles and friction between the thrombus and the vessel wall even more.

On the other hand, curved vessels were not associated with a significantly increased number of periprocedural complications, such as thrombus dispersion, vessel perforation, or intracranial hemorrhage. Therefore, patients with curved vessels apparently do not have an increased periprocedural risk profile if MT is performed.

Regarding different devices, this study did not show any significant superiority of any of the used stent retrievers compared with other models in patients with curved vessels, even though more recently developed stent retrievers have been designed to provide more stability when being retracted around vessel curves and therefore are supposed to avoid the "tapering" phenomenon. ${ }^{12,13}$

Although vessel curvature significantly influences the results of MT by using stent retrievers, TICI $2 \mathrm{~b} / 3$ was still achieved in one-half of patients presenting with curved vessels with proximal occlusions and approximately one-third of patients with curved vessels and distal occlusions. Therefore, patients should never be excluded from MT solely on the basis of their vessel angles. However, further development and individualized selection of retrieval devices regarding vessel anatomy may improve the recanalization success in patients with curved vessels. For instance, more bendable devices with a design advanced from the classic stent configuration may perform differently.

Additionally, alternative techniques such as the forced-suction approach, as described in a recent trial, should be assessed in patients with curved vessels because recanalization rates of up to $95 \%$ have been reported for this technique. ${ }^{26}$ A number of studies on suction-based approaches have been published recently, showing inconsistent results; however, none of these studies analyzed vessel anatomy as a factor influencing recanalization success. ${ }^{27-29}$

Aortic and cervical vessel anatomy may influence recanalization results as well. Especially, older patients with a history of hypertension present with elongated arteries that may impede catheterization and lengthen the procedure duration. Still, no significant influences of age and procedure time on TICI scores were found. Similarly, neither the type of anesthetic nor the NIHSS score at admission significantly influenced the recanalization results. Therefore, we conclude, in this retrospective analysis, that 
any possible influence from those parameters on technical results of MT was secondary.

This study focused on intracranial vessel anatomy as 1 factor influencing MT results. It is plausible that other factors (ie, thrombus composition) have a significant influence as well. These should be considered and analyzed in further prospective studies.

Overall, MT significantly improved recanalization rates and clinical outcome in patients with acute ischemic stroke caused by large-vessel occlusion by using stent retrievers. ${ }^{5-11}$ However, in approximately $10 \%-25 \%$ of patients, a successful recanalization (TICI 2b/3) could not be achieved. ${ }^{5-14}$ So far, not many factors are fully understood in determining the recanalization results of these patients. One significant factor is vessel anatomy, as this retrospective study showed.

\section{CONCLUSIONS}

In this study, the influence of vessel anatomy, assessed in anteroposterior angiograms, on recanalization results by using stent retrievers in patients with acute ischemic stroke after large-vessel occlusion was described for the first time. It was shown that patients with unsuccessful recanalization (TICI 0-2a) had significantly stronger curved carotid Ts, distal M1 segments, and/or proximal M2 segments than patients with successful recanalization results (TICI 2b/3). Further experimental work is needed to understand the causality of this finding and to develop and analyze new treatment strategies and devices for this patient subgroup.

\section{ACKNOWLEDGMENTS}

The authors thank Sebastian Obermeyer for the illustrations and Alexander Hapfelmeier for statistical advice.

Disclosures: Sascha Prothmann—UNRELATED: Consultancy: proctoring for Phenox; Employment: consultant at Klinikum rechts der Isar, Munich.

\section{REFERENCES}

1. Seshadri S, Wolf PA. Lifetime risk of stroke and dementia: current concepts, and estimates from the Framingham Study. Lancet Neurol 2007;6:1106-14

2. Mori E, Yoneda $\mathrm{Y}$, Tabuchi $\mathrm{M}$, et al. Intravenous recombinant tissue plasminogen activator in acute carotid artery territory stroke. $\mathrm{Neu}$ rology 1992;42:976-82

3. Saqqur M, Uchino K, Demchuk AM, et al; CLOTBUST Investigators. Site of arterial occlusion identified by transcranial Doppler predicts the response to intravenous thrombolysis for stroke. Stroke 2007;38:948-54

4. Smith WS, Lev MH, English JD, et al. Significance of large vessel intracranial occlusion causing acute ischemic stroke and TIA. Stroke 2009;40:3834-40

5. Dávalos A, Pereira VM, Chapot R, et al; Solitaire Group. Retrospective multicenter study of Solitaire FR for revascularization in the treatment of acute ischemic stroke. Stroke 2012;43:2699-705

6. Nogueira RG, Lutsep HL, Gupta R, et al; TREVO 2 Trialists. Trevo versus Merci retrievers for thrombectomy revascularisation of large vessel occlusions in acute ischaemic stroke (TREVO 2): a randomised trial. Lancet 2012;380:1231-40

7. Pereira VM, Gralla J, Davalos A, et al. Prospective, multicenter, single-arm study of mechanical thrombectomy using Solitaire flow restoration in acute ischemic stroke. Stroke 2013;44:2802-07

8. Saver JL, Jahan R, Levy EI, et al; SWIFT Trialists. Solitaire flow restoration device versus the Merci retriever in patients with acute ischaemic stroke (SWIFT): a randomised, parallel-group, non-inferiority trial. Lancet 2012;380:1241-49
9. Yoon YH, Yoon W, Jung MY, et al. Outcome of mechanical thrombectomy with Solitaire stent as first-line intra-arterial treatment in intracranial internal carotid artery occlusion. Neuroradiology 2013; 55:999-1005

10. Zaidat OO, Castonguay AC, Gupta R, et al. North American Solitaire Stent Retriever Acute Stroke registry: post-marketing revascularization and clinical outcome results. J Neurointerv Surg 2014; 6:584-88

11. Broussalis E, Trinka E, Hitzl W, et al. Comparison of stent-retriever devices versus the Merci retriever for endovascular treatment of acute stroke. AJNR Am J Neuroradiol 2013;34:366-72

12. Kurre W, Aguilar-Pérez M, Schmid E, et al. Clinical experience with the pREset stent retriever for the treatment of acute ischemic stroke-a review of 271 consecutive cases. Neuroradiology 2014;56:397-403

13. Schwaiger BJ, Kober F, Gersing AS, et al. The pREset stent retriever for endovascular treatment of stroke caused by MCA occlusion: safety and clinical outcome. Clin Neuroradiol 2014 Aug 12. [Epub ahead of print]

14. Jansen O, Macho JM, Killer-Oberpfalzer M, et al; TREVO Study Group. Neurothrombectomy for the treatment of acute ischemic stroke: results from the TREVO study. Cerebrovasc Dis 2013; 36:218-25

15. Yuki I, Kan I, Vinters HV, et al. The impact of thromboemboli histology on the performance of a mechanical thrombectomy device. AJNR Am J Neuroradiol 2012;33:643-48

16. Gralla J, Burkhardt $M$, Schroth $G$, et al. Occlusion length is a crucial determinant of efficiency and complication rate in thrombectomy for acute ischemic stroke. AJNR Am J Neuroradiol 2008;29:247-52

17. Jindal G, Miller T, Shivashankar R, et al. Relationship of thrombus length to number of stent retrievals, revascularization, and outcomes in acute ischemic stroke. J Vasc Interv Radiol 2014;25:1549-57

18. Behme D, Kowoll A, Weber W, et al. M1 is not M1 in ischemic stroke: the disability-free survival after mechanical thrombectomy differs significantly between proximal and distal occlusions of the middle cerebral artery M1 segment. J Neurointerv Surg 2014 Jun 24. [Epub ahead of print]

19. Zhu L, Liebeskind DS, Jahan R, et al. Thrombus branching and vessel curvature are important determinants of middle cerebral artery trunk recanalization with Merci thrombectomy devices. Stroke 2012;43:787-92

20. Veltkamp R. Akuttherapie des ischämischen Schlaganfalls. In: Diener HC, Weimar C, eds. Leitlinien für Diagnostik und Therapie in der Neurologie. 5th ed. Stuttgart: Thieme; 2012:307-23

21. Fisher M, Albers GW. Advanced imaging to extend the therapeutic time window of acute ischemic stroke. Ann Neurol 2013;73:4-9

22. Parsons M, Spratt N, Bivard A, et al. A randomized trial of tenecteplase versus alteplase for acute ischemic stroke. $N$ Engl J Med 2012;366:1099-107

23. Burton KR, Dhanoa D, Aviv RI, et al. Perfusion CT for selecting patients with acute ischemic stroke for intravenous thrombolytic therapy. Radiology 2015;274:103-14

24. Berger C, Fiorelli M, Steiner T, et al. Hemorrhagic transformation of ischemic brain tissue: asymptomatic or symptomatic? Stroke 2001; 32:1330-35

25. Youden WJ. Index for rating diagnostic tests. Cancer 1950;3:32-35

26. Turk AS, Frei D, Fiorella D, et al. ADAPT FAST study: a direct aspiration first pass technique for acute stroke thrombectomy. J Neurointerv Surg 2014;6:260-64

27. Kim SK, Yoon W, Moon SM, et al. Outcomes of manual aspiration thrombectomy for acute ischemic stroke refractory to stent-based thrombectomy. J Neurointerv Surg 2014 May 7. [Epub ahead of print]

28. Kang DH, Kim YW, Hwang YH, et al. Switching strategy for mechanical thrombectomy of acute large vessel occlusion in the anterior circulation. Stroke 2013;44:3577-79

29. Kass-Hout T, Kass-Hout O, Sun CH, et al. Clinical, angiographic and radiographic outcome differences among mechanical thrombectomy devices: initial experience of a large-volume center. J Neurointerv Surg 2015;7:176-81 